\title{
Identificazione rapida di mutazioni associate a farmaco-resistenza in ceppi di citomegalovirus umano mediante nPCR-RFLP
}

\author{
Maria Cristina Medici, Monica Martinelli, Annalisa Aloisi, Laura Anna Abelli, \\ Giuseppe Dettori, Carlo Chezzi
}

Sezione di Microbiologia, Dipartimento di Patologia e Medicina di Laboratorio, Università degli Studi di Parma

Key words: human cytomegalovirus (HCMV), nPCR-RFLP, ganciclovir resistance, transplant recipients Rapid identification and detection of ganciclovir-resistant human cytomegalovirus strains by
nPCR-RFLP

\section{SUMMARY}

We developed a nested-PCR followed by restriction fragment length polymorphism (RFLP) for the detection of human cytomegalovirus (HCMV) UL97 M460V/I, H520Q, C592Q, A594V, L595S/F and C603W mutations associated to ganciclovir (GCV) resistance. The method uses five primer pairs and seven enzymes already published and newly combined. The detection limit of nPCR was assessed in a single serial dilution assay to be about 0.13 PFU/reaction. Expected restriction fragment patterns were obtained by nPCR-RFLP on either wild-type reference strains or strains and sequences of HCMV containing mutations. Then the nPCR-RFLP was used on 24 sera/plasma belonging to 22 transplant recipients (kidney, bone marrow, or kidney-pancreas): I 3 subjects never treated with GCV (control group) and 9 subjects treated with GCV oral profilaxis (study group). All codons detected from the control group (six in 8 cases and four in I case) were identified as wild-type. All codons detected from the study group (six in 6 cases, three in 2 cases, and four in the second sample of I case whose first sample was negative by $\mathrm{nPCR}$ ) were wild-type except one, which showed a restriction pattern referring to M460V and/or M460I ATA-codified, definitively proved to be M460V by sequence analysis. This was the case of a renal transplant recipient at the end of profilaxis.

In conclusion, the procedure seems to be quite sensitive and specific as well as able to detect mixed population of mutants or mutants and wild-type. It could represent a good tool in monitoring the emergence of HCMV mutants in renal transplant recipients treated with GCV.

\section{INTRODUZIONE}

L'infezione da citomegalovirus umano (HCMV) è una delle cause più frequenti di malattia virale in soggetti sottoposti a trapianto d'organo o di midollo. Ganciclovir (GCV) e valganciclovir (VGCV) rappresentano attualmente i farmaci più utilizzati nel trattamento sistemico e/o nella profilassi dell'infezione/malattia da HCMV. Per l'attivazione di tali farmaci sono necessarie fosforilazioni ad opera di una protein kinasi virale codificata dal gene UL97 e di chinasi cellulari (5).

È noto che talora la terapia con GCV o VGCV può fallire per l'insorgenza di mutazioni sul gene UL97 di HCMV. È stato ipotizzato che la pratica di un prolungato periodo di profilassi orale con tali farmaci, da un lato, e l'uso di più intensive strategie immunosoppressive, dall'altro, potrebbero contribuire all'insorgenza di varianti di HCMV GCV-resistenti $(1,7,9)$.

La dimostrazione che la resistenza fenotipica è associata a specifiche mutazioni ha aumentato l'interesse verso lo sviluppo di metodi genotipici più sensibili, specifici e rapidi rispetto al saggio fenotipico di riferimento basato sulla inibizione dell'infettività virale in vitro. Procedimenti basati sull'analisi del polimorfismo della lunghezza dei frammenti di restrizione (RFLP) previa amplificazione mediante PCR di regioni sul gene UL97 di HCMV hanno consentito di identificare le più frequenti mutazioni associate a GCV-resistenza (5).

Ad oggi sono stati condotti solo pochi studi sull'incidenza di ceppi di HCMV farmaco-resistenti su coorti di pazienti sottoposti a trapianto $(1,7,8,11)$. Obiettivo di questo studio è stato innanzitutto quello di sviluppare un procedimento di RFLP previa amplificazione mediante PCR di tipo nested (nPCR-RFLP) per rivelare e identificare le mutazioni M460V/I, H520Q, C592G, A594V, $\mathrm{L} 595 \mathrm{~F} / \mathrm{S}$ e $\mathrm{C} 603 \mathrm{~W}$ che più frequentemente insorgono sul gene UL97 di HCMV e che sono associate a GCV-resistenza.

Ulteriore obiettivo è stato quello di impiegare il procedimento di nPCR-RFLP su campioni clinici appartenenti a soggetti sottoposti a trapianto di 
rene o di midollo osseo e a profilassi orale con GCV nel tentativo di valutare la presenza di ceppi di HCMV farmaco-resistenti in questa categoria di soggetti.

\section{MATERIALI E METODI}

Ceppi di HCMV "wild-type" di riferimento

Sono stati impiegati i ceppi di riferimento HCMVAD169 (ATCC n ${ }^{\circ}$ VR538) e -Towne (ATCC n ${ }^{\circ}$ VR977) in dotazione presso l'unità operativa di Virologia del dipartimento di Patologia e Medicina di Laboratorio dell'Azienda ospedaliero - universitaria di Parma, conservati congelati a $-80^{\circ} \mathrm{C}$ in forma di sospensione (625.000 PFU $/ \mathrm{ml} \mathrm{e}$ $450.000 \mathrm{PFU} / \mathrm{ml}$, rispettivamente).

Ceppi e sequenze di HCMV di riferimento contenenti mutazioni associate a farmaco-resistenza Sono stati impiegati ceppi di HCMV contenuti in 4 sospensioni virali ("Strain pp6": HCMV UL97M460V e HCMV UL97-M460I; "Isolat 1": HCMV UL97-M460V; "Isolat 2": HCMV UL97M460, HCMV UL97-M460V e HCMV UL97M460I; "Isolat 6": HCMV UL97-L595S), gentilmente fornite dal dottor Klaus Hamprecht del dipartimento di Virologia Medica e di Epidemiologia delle Malattie Virali dell'Ospedale universitario di Tübingen, Germania. Inoltre sono state utilizzate sequenze amplificate dalla regione compresa tra i nucleotidi 784 e 1871 del gene UL97 di 3 ceppi ricombinanti di HCMV-AD169 contenenti le mutazioni UL97-M460V, UL97A594V e UL97-L595S, rispettivamente, cortesemente forniti in forma liofila dal professor Sunwen Chou della Divisione di Malattie Infettive del Veterans Affairs Medical Center-Health and Science University di Portland, Oregon.

Ciascun campione liofilo è stato reidratato con $500 \mu 1$ di tampone Tris-EDTA ( $\mathrm{pH} 8,2$ ).

Un'aliquota di $1 \mu 1$ di ciascun campione reidratato è stata quindi sottoposta a ulteriore amplificazione genica mediante la coppia di primer interni prevista nell'nPCR-RFLP per l'identificazione della mutazione in esso contenuta.

\section{Campioni clinici e soggetti}

Sono stati esaminati 24 campioni di siero/plasma, appartenenti a 22 soggetti sottoposti a trapianto d'organo o midollo ( 15 di rene, 6 di midollo e 1 di rene-pancreas) e ricoverati presso i reparti di Nefrologia e di Ematologia e Centro Trapianti di Midollo Osseo dell'Azienda ospedaliero - universitaria di Parma nel periodo 2002-2004, risultati positivi per HCMV-DNA alle indagini qualitative e/o quantitative condotte a scopo diagnostico presso l'u.o. di Virologia della stessa Azienda e conservati congelati $\mathrm{a}-20^{\circ} \mathrm{C}$. Questi soggetti erano risul- tati positivi anche per l'antigene HCMV-pp65 nei leucociti polimorfonucleati (PMNL) del sangue periferico. I 22 soggetti comprendevano un gruppo di 13 soggetti mai trattati con GCV (gruppo di controllo) e un gruppo di 9 soggetti sottoposti a profilassi orale con questo farmaco (gruppo di studio), dei quali al momento del prelievo 6 erano ancora in corso di trattamento, 2 al termine e 1 a circa due anni dalla fine del trattamento. Quest'ultimo soggetto presentava febbre ed epatite mentre tutti $\mathrm{i}$ rimanenti erano asintomatici. Inoltre di 2 soggetti del gruppo di studio erano disponibili 2 campioni prelevati a distanza di 3 e 2 mesi, rispettivamente.

\section{Estrazione dell'acido nucleico}

Il DNA virale è stato estratto in modo rapido mediante idrocloruro di guanidina ("EXTRAgen kit", Amplimedical) da $300 \mu 1$ di siero/plasma o sospensione virale e risospeso in $10 \mu \mathrm{l}$ di acqua priva di nucleasi (concentrazione finale $25 \mathrm{X}$ ).

\section{PCR qualitativa, PCR quantitativa e antigenemia}

La ricerca del DNA di HCMV è stata condotta sui campioni di siero/plasma mediante nPCR utilizzando il saggio qualitativo "CMV early-kit" (Amplimedical).

La determinazione quantitativa del DNA virale è stata condotta mediante il saggio semiautomatizzato "COBAS Amplicor CMV Monitor Test" (Roche Diagnostics).

La ricerca dell'antigene HCMV-pp65 è stata condotta mediante immunofluorescenza indiretta con anticorpo monoclonale specifico (Argene Biosoft) su due preparati di $2 \times 10^{5}$ PMNL del sangue periferico. Il numero di PMNL positivi per pp65 (colorazione nucleare verde mela brillante) è stato contato e registrato.

\section{nPCR-RFLP}

Per l'identificazione delle mutazioni M460V/I, H520Q, C592G, A594V, L595F/S e C603W associate a GCV-resistenza è stato allestito un procedimento di nPCR-RFLP utilizzando una nuova combinazione di 5 coppie di primer e di 7 enzimi di restrizione, già descritti in letteratura $(2,3,4,6$, 10), per l'amplificazione e la digestione di 4 frammenti genomici. I primer e gli enzimi unitamente alle dimensioni dei prodotti di amplificazione e dei frammenti di restrizione, relativi sia alle sequenze mutate sia a quelle "wild-type", sono mostrati in tabella 1 . I primer scelti sono stati selezionati sulla base di informazioni bibliografiche $(2,3,4,6,10)$ e dal confronto delle sequenze dei ceppi di HCMV depositate in Genbank mediante software BLAST2.

In breve, $10 \mu \mathrm{l}$ di DNA estratto sono stati aggiunti 
ad una miscela contenente 1X GeneAmp PCR Buffer II (Applied Biosystems), 1,5 mM $\mathrm{MgCl}_{2}$ (Applied Biosystems), $200 \mu \mathrm{M}$ di ciascun nucleotide trifosfato (Amersham Bioscience), 0,5 $\mu \mathrm{M}$ di ciascun primer esterno (CPT0 e CPT2096), 1,25 U di Ampli Taq Gold (Applied Biosystems) in un volume finale di $50 \mu \mathrm{l}$. Un'aliquota di $1 \mu 1$ del prodotto di prima amplificazione è stata aggiunta a 4 diverse miscele per la seconda amplificazione quali/quantitativamente analoghe a quella della prima eccetto i primer esterni che sono stati sostituiti dalle coppie di primer interni (CPT1088/CPT1619, CPT1713/CPT1830M, CPT1088/CPT1587M e 595F/595R, rispettivamente). Di queste le due coppie CPT1088/CPT1587M e $595 \mathrm{~F} / 595 \mathrm{R}$ sono state impiegate per la prima volta come primer di seconda amplificazione in una reazione nested. Entrambe le fasi di amplificazione sono state condotte nelle stesse condizioni termodinamiche in termociclatore automatico (GeneAmp PCR System 9700, Applied Biosystems) secondo il protocollo di Chou e coll. (3).

I prodotti di amplificazione sono stati sottoposti ad elettroforesi in gel di agarosio (Biorad) al 2.5\% in tris-acetato-EDTA (TAE) 1X colorato con bromuro di etidio e il tracciato elettroforetico è stato visualizzato mediante transilluminatore UV dotato di sistema computerizzato per l'acquisizione di immagini (Gel Analyzer, Clonit).

Per l'RFLP sono stati utilizzati i seguenti enzimi di restrizione: NlaIII (New England Biolabs), CfoI (Roche Applied Science), TaqI (Roche Applied Science), Tru9I (Roche Applied Science), AluI (Roche Applied Science), FseI (New England Biolabs) e AvaII (Roche Applied Science).

In breve, la digestione enzimatica è stata condotta aggiungendo $17 \mu \mathrm{l}$ di prodotto di seconda amplificazione del frammento di interesse ad una miscela contenente $10 \mathrm{U}$ di enzima di restrizione e il relativo buffer (1X) in un volume finale di $20 \mu$ l.

Le condizioni termiche di attività enzimatica caratteristiche di ciascun enzima di restrizione sono state determinate sulla base delle informazioni fornite dal produttore.

I prodotti di amplificazione di $118 \mathrm{pb}$, digeriti dagli enzimi $C f o$ I, TaqI e Tru9I, sono stati sottoposti a corsa elettroforetica $(120 \mathrm{~V}$ per circa 60 min) in gel di agarosio ad alta risoluzione Metaphor al 3\% (Cambrex Bio Science) in trisborato-EDTA 1X (Invitrogen) e quelli di $532 \mathrm{pb}$, $501 \mathrm{pb}$ e $189 \mathrm{pb}$, digeriti dagli enzimi NlaIII, Tru9I, AluI, FseI e AvaII, in gel di agarosio al $3,5 \%$ (BioRad) in TAE.

Dal momento che il codone UL97-460 può essere interessato da tre distinti eventi mutazionali (ATG codificante per metionina $\rightarrow$ GTG codificante per valina, $\rightarrow$ ATA o $\rightarrow$ ATT codificanti per isoleuci- na) che conferiscono GCV-resistenza e che non sono distinguibili sulla base dell'impiego del solo enzima NlaIII (sito di taglio CATG), nel saggio è stato previsto l'utilizzo dell'enzima Tru9I (sito di taglio CATT). Quest'ultimo consente di escludere la presenza della mutazione M460I ATT-codificata attraverso la mancata digestione del prodotto di amplificazione risultato contenere il codone 460 mutato alla digestione con NlaIII. Tru9I rappresenta l'isoschizomero dell'enzima MseI suggerito da Eckle e coll., 2003 (4).

\section{Sequenziamento}

Il sequenziamento è stato condotto su entrambi i filamenti del prodotto di amplificazione, purificato mediante saggio "Qiaquick Gel Extraction" (QIAGEN), in sequenziatore automatico 3730 DNA Analyzer (Applied Biosystems) secondo la chimica "dye terminator" utilizzando le stesse coppie di primer interni della nPCR. Le sequenze sono state confrontate con quelle dei ceppi di riferimento depositate in Genbank (numero di accesso X17403 per HCMV-AD169 e AY315197 per HCMV-Towne) mediante software BLAST2.

\section{RISULTATI}

\section{Saggio di amplificazione genica - Sensibilità analitica}

Al fine di valutare l'efficacia del procedimento di nPCR allestito, esso è stato innanzitutto provato sui ceppi di riferimento HCMV-AD169 e HCMVTowne. All'elettroforesi dei prodotti delle 4 diverse amplificazioni tutti i ceppi hanno dato bande di amplificazione delle dimensioni attese $(532 \mathrm{pb}$, $501 \mathrm{pb}, 189 \mathrm{pb}$ e $118 \mathrm{pb}$, rispettivamente). In secondo luogo, al fine di valutare la sensibilità analitica del metodo, il procedimento è stato provato su diluizioni seriali in base $10\left(10^{-1}-10^{-9}\right)$ delle sospensioni a concentrazione nota di HCMV-AD169 e HCMV-Towne, rispettivamente. Una chiara banda delle dimensioni attese per tutti i 4 prodotti di amplificazione è stata osservata all'elettroforesi degli ampliconi di entrambi i ceppi fino alla diluizione $10^{-6}$ (pari a 0,156 PFU/reazione per HCMV-AD169 e 0,112 PFU/reazione per HCMV-Towne). La diluizione $10^{-3}$ di entrambi i ceppi, contenente $156 \mathrm{PFU} /$ reazione per HCMV-AD169 e 112 PFU/reazione per HCMV-Towne, è stata scelta come controllo positivo di reazione per le prove successive.

Saggio di RFLP su ceppi e sequenze contenenti mutazioni a confronto con ceppi "wild-type" di riferimento.

L'nPCR-RFLP, impiegata sui ceppi di HCMV e sulle sequenze di DNA virale di riferimento contenenti in diversa misura i codoni mutati M460V, 
M460I, A594V e L595S, ha generato profili di restrizione riferibili alle mutazioni di interesse secondo l'atteso, a confronto con quelli ottenuti dai ceppi non mutati di riferimento.

In particolare, l'impiego della combinazione degli enzimi NlaIII e Tru9I sulle sospensioni virali "Isolat 1" e "Strain pp6" contenenti la mutazione M460V da sola e una popolazione mista di due ceppi mutati di HCMV, rispettivamente, ha consentito di distinguere le mutazioni M460V e M460I (ATT codificata) presenti negli stipiti virali in sospensione (Figura 1). Infatti, da un lato, NlaIII ha generato sia da "Isolat 1" che da "Strain pp6" due frammenti di restrizione di 324 pb e 168 pb, rivelando la perdita di un sito di taglio dovuta alla presenza di una mutazione generica al codone $460 \mathrm{e}$, dall'altro, Tru9I ha generato da "Isolat 1" un frammento di $532 \mathrm{pb}$ (non digerito), dovuto all'assenza della mutazione M460I (ATT), tale da poter assumere la presenza attesa della mutazione M460V (GTG), e da "Strain pp6" frammenti di 292 pb e $240 \mathrm{pb}$, oltre a quello non digerito di $532 \mathrm{pb}$, rivelando l'acquisizione di un sito di taglio dovuto alla mutazione M460I (ATT), in accordo con la presenza nel campione di un secondo ceppo virale mutato oltre a quello con mutazione M460V.

Inoltre, il saggio condotto mediante NlaIII e Tru9I sulla sospensione virale "Isolat 2" ha rivelato la

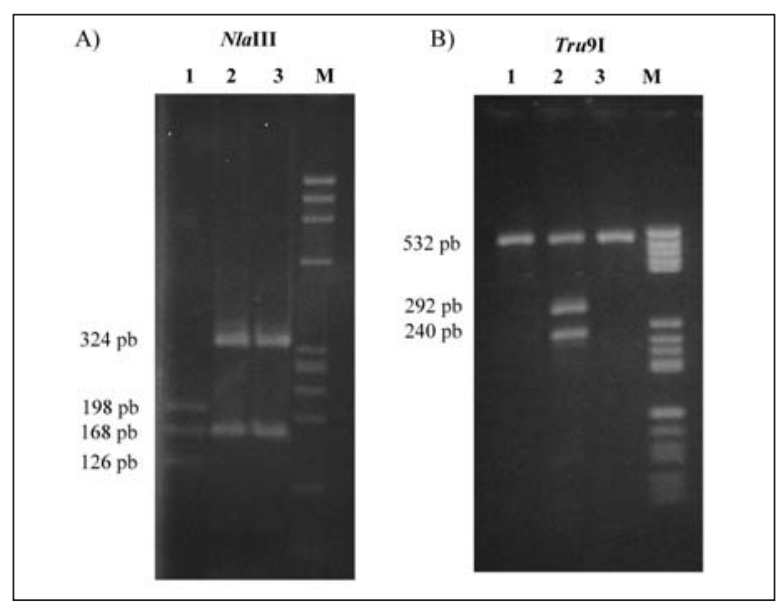

Figura I.: Analisi dei profili di restrizione per la distinzione delle mutazioni M460V e M460I (ATT) sul gene UL97 di HCMV in ceppi di riferimento. A) Digestione con Nlalll. Posizione I, profilo di ceppo "wild-type" (HCMV-ADI69); posizioni 2 e 3, profili di ceppi mutati ("Strain pp6" e "Isolat I", rispettivamente: la presenza di M460V e/o M460I possono essere desunte); $M$, indicatore di pesi molecolari. B) Digestione con Tru9l a confronto con Nlalll. Posizione I, profilo di ceppo "wild-type" (HCMV-AD I69); posizione 2, profilo riferibile a una miscela di ceppi mutati ("Strain pp6": M460I (ATT) è presente, M460V può essere desunta e M460l (ATA) non può essere esclusa); posizione 3, profilo riferibile a ceppo mutato ("Isolat I": M460I (ATT) è esclusa e M460V può essere desunta); $M$, indicatore di pesi molecolari. presenza di almeno due ceppi virali (Figura 2): uno "wild-type", identificato dai frammenti di 198 pb, 168 pb e 126 pb ottenuti con NlaIII e da quello di $532 \mathrm{pb}$ ottenuto con Tru9I, e uno mutato (M460I - ATT codificata), identificato dai frammenti di 324 pb e 168 pb ottenuti con NlaIII e da quelli di 292 pb e 240 pb ottenuti con Tru9I. La presenza dei frammenti riferibili a sequenze "wild-type" e mutate nei profili di restrizione ottenuti dalla digestione con l'uno e con l'altro enzima è risultata anche compatibile con la presenza attesa nel campione di un terzo ceppo virale M460V mutato (digestione con NlaIII: frammenti di 324 pb e 168 pb; digestione con Tru9I: frammento di $532 \mathrm{pb}$ ).

\section{Saggio su campioni clinici}

L'nPCR-RFLP condotta sui 22 soggetti sottoposti a trapianto d'organo o di midollo, nell' ambito dei 13 soggetti di controllo, ha identificato 6 codoni in 8 casi $(61.5 \%), 4$ codoni in un caso $(7.7 \%) \mathrm{e}$ nessun codone in 4 casi $(30.8 \%)$ (Tabella II). Tutti i codoni identificati sono risultati "wild-type". In 4 casi l'RFLP non è stata condotta per assenza del prodotto di amplificazione, in un caso il campione conteneva $2.2 \times 10^{3}$ copie genomiche $/ \mathrm{ml}$, in due casi i campioni contenevano livelli di DNA virale al di sotto del limite di rilevabilità del sag-

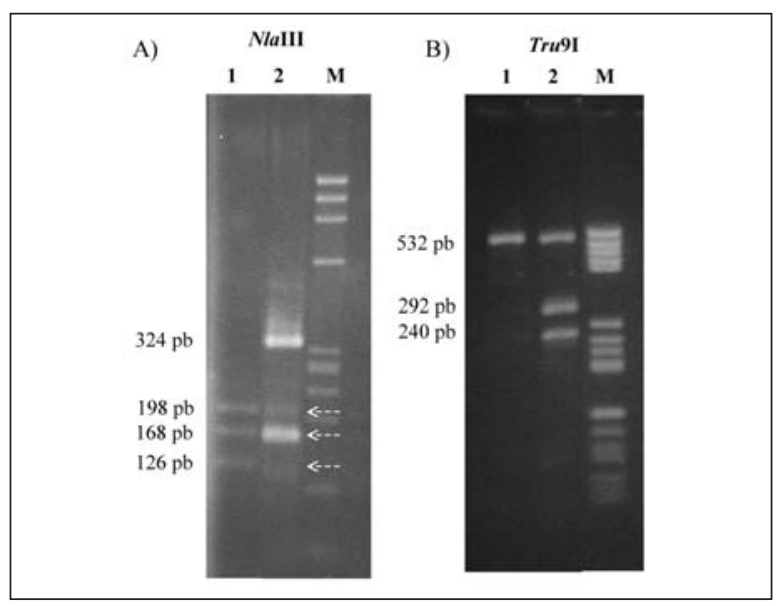

Figura II.: Analisi dei profili di restrizione per la rivelazione di popolazioni virali miste di HCMV in un ceppo di riferimento.A) Digestione con Nlalll. Posizione I, profilo di ceppo "wild-type" (HCMV-AD I69); posizione 2, profilo riferibile a una miscela di ceppi "wild-type", indicato dalle frecce, e mutato ("Isolat 2": M460 è presente e M460V e/o M460l possono essere desunte); $M$, indicatore di pesi molecolari. B) Digestione con Tru9l a confronto con Nlalll. Posizione I, profilo di ceppo "wild-type" (HCMV-ADI69); posizione 2, profilo riferibile a una miscela di ceppi "wild-type" e mutati ("Isolat 2": M460 e M460I (ATT) sono presenti, M460V può essere desunta e M460I (ATA) non può essere esclusa); $M$, indicatore di pesi molecolari. 
Tabella I. Primer ed enzimi di restrizione utilizzati per l'identificazione delle mutazioni M460V, M460l, H520Q, C592G, C603W, A594V, L595S e L595F sul gene UL97di HCMV.

\begin{tabular}{|c|c|c|c|c|c|c|c|c|}
\hline \multicolumn{2}{|l|}{ I Amplificazione } & \multicolumn{2}{|l|}{ II Amplificazione } & \multicolumn{4}{|l|}{ RFLP } & \multirow{3}{*}{$\begin{array}{l}\text { Riferimento } \\
\text { - bibliografico }\end{array}$} \\
\hline \multirow{2}{*}{$\begin{array}{l}\text { Coppia } \\
\text { di primer } \\
(+/-)\end{array}$} & \multirow{2}{*}{$\begin{array}{l}\text { Dimensione } \\
\text { amplicone } \\
\text { (pb) }\end{array}$} & \multirow{2}{*}{$\begin{array}{l}\text { Coppia } \\
\text { di primer } \\
(+/-)\end{array}$} & \multirow{2}{*}{$\begin{array}{l}\text { Dimensione } \\
\text { amplicone } \\
\text { (pb) }\end{array}$} & \multirow[b]{2}{*}{ Enzima } & \multirow{2}{*}{$\begin{array}{l}\text { Mutazione } \\
\text { rivelabile }\end{array}$} & \multicolumn{2}{|c|}{ Dimensioni frammento (pb) } & \\
\hline & & & & & & $\begin{array}{l}\text { Sequenza } \\
\text { wt }\end{array}$ & $\begin{array}{l}\text { Sequenza } \\
\text { mutata }\end{array}$ & \\
\hline \multirow{6}{*}{ СРТО/СРТ2096 } & \multirow{6}{*}{2232} & CPTI088/CPTI619 & 532 & $\begin{array}{l}\text { NlallI } \\
\text { Tru9| }\end{array}$ & $\begin{array}{l}\text { M460V/I } \\
\text { M460I }\end{array}$ & $\begin{array}{l}198,168,126,(40) \\
532\end{array}$ & $\begin{array}{l}324,168,(40) \\
292,240\end{array}$ & 2,4 \\
\hline & & CPTI088/CPTI587M & 501 & Alul & H520Q & $295,189,(17)$ & $268,189,27,(17)$ & 2,6 \\
\hline & & $595 \mathrm{~F} / 595 \mathrm{R}$ & 189 & $\begin{array}{l}\text { Fsel } \\
\text { Avall }\end{array}$ & $\begin{array}{l}\text { C592G } \\
\text { C603W }\end{array}$ & $\begin{array}{l}189 \\
173,(16)\end{array}$ & $\begin{array}{l}106,83 \\
104,69,(16)\end{array}$ & 2,10 \\
\hline & & & & Cfol & A594V & $50,83,(18),(12)$ & $62,38,(18)$ & \\
\hline & & CPTI7I3/CPTI830M & 118 & Toql & L595S & $99,(19)$ & $71,28,(19)$ & 2,3 \\
\hline & & & & Tru9l & L595F & 76,42 & $46,42,30$ & \\
\hline
\end{tabular}

$(+/-)$ : senso/anti-senso

wt: "wild-type"

():frammenti non rivelabili mediante elettroforesi

Tabella 2. Identificazione mediante nPCR-RFLP delle mutazioni M460V, M460I, H520Q, C592G, C603W,A594V, L595S e L595F sul gene UL97 di HCMV in ceppi rivelati in 22 soggetti viremici sottoposti a trapianto d'organo o di midollo, 13 appartenenti al gruppo di controllo e 9 al gruppo di studio, in relazione alla quantità di HCMV-DNA e di leucociti polimorfonucleati (PMNL) positivi per HCMVpp65. - assenza di mutazioni.

\begin{tabular}{|c|c|c|c|c|c|c|c|c|c|c|c|c|}
\hline \multicolumn{7}{|c|}{ Gruppo di controllo } & \multicolumn{6}{|c|}{ Gruppo di studio } \\
\hline $\mathbf{N}^{\circ}$ & $\begin{array}{l}\text { Iniziali } \\
\text { soggetto }\end{array}$ & $\begin{array}{l}\text { Data } \\
\text { oprelievo }\end{array}$ & $\begin{array}{l}\text { DNA } \\
\text { (copie/ml) }\end{array}$ & $\begin{array}{l}N^{\circ} P M N L \\
\text { pp65 } \\
\text { pos } / 2 \times 10^{5}\end{array}$ & $\begin{array}{l}\mathbf{N}^{\circ} \text { codoni } \\
\text { identificati }\end{array}$ & $\begin{array}{l}\text { Mutazioni } \\
\text { su UL97 }\end{array}$ & $\begin{array}{c}\text { Iniziali } \\
\mathrm{N}^{\circ} \text { soggetto }\end{array}$ & $\begin{array}{l}\text { Data } \\
\text { prelievo }\end{array}$ & $\begin{array}{l}\text { DNA } \\
\text { (copie/ml) }\end{array}$ & $\begin{array}{l}N^{\circ} \text { PMNL } \\
\text { Pp65 } \\
\text { pos } / 2 \times 10^{5}\end{array}$ & $\begin{array}{l}\mathbf{N}^{\circ} \text { codoni } \\
\text { identificati }\end{array}$ & $\begin{array}{l}\text { Mutazioni } \\
\text { su UL97 }\end{array}$ \\
\hline 1 & V. L. & $17 / 01 / 03$ & $8,33 \times 10^{4}$ & $>500$ & 6 & - & I V. G.F. & $03 / 11 / 03$ & $1,65 \times 10^{3}$ & 17 & 6 & - \\
\hline 2 & B. P. & $21 / 06 / 04$ & "target low"* & 1 & 6 & - & 2 Z.V. & $27 / 10 / 03$ & $8,32 \times 10^{3}$ & 4 & 6 & - \\
\hline 3 & C. M. & $21 / 10 / 04$ & $1,25 \times 10^{3}$ & 100 & 6 & - & 3 L. I. & $21 / 10 / 04$ & $3,32 \times 10^{3}$ & 400 & 6 & M460V \\
\hline 4 & B. C. & $04 / 02 / 05$ & $4,86 \times 10^{2}$ & 63 & 6 & - & 4 T. M. & $03 / 11 / 03$ & $9,87 \times 10^{3}$ & 65 & 6 & - \\
\hline 5 & R. L. & $30 / 12 / 04$ & $<600$ & 21 & 6 & - & 5 B. S. & $14 / 11 / 02$ & $1,21 \times 10^{3}$ & 30 & 6 & - \\
\hline 6 & R. C. & $13 /|| / 03$ & $1,4 \times 10^{4}$ & 50 & 6 & - & 6 B. I. & $27 / 10 / 03$ & $1,45 \times 10^{3}$ & 18 & 6 & - \\
\hline 7 & F. A & $10 / 05 / 04$ & $<600$ & 4 & 6 & - & & $22 / 12 / 03$ & $2,17 \times 10^{4}$ & 5 & 6 & $\cdot$ \\
\hline 8 & V. I. & $12 / 12 / 02$ & $1,51 \times 10^{3}$ & 70 & 6 & - & 7 P. E. & $09 / 01 / 03$ & "target low" & 127 & 3 & - \\
\hline 9 & B. A. & $14 / 03 / 02$ & $<600$ & 10 & 4 & - & 8 F.M. & I7/07/03 & n.d. & 32 & 3 & - \\
\hline 10 & C. G. & $02 / 10 / 03$ & $2,2 \times 10^{3}$ & 24 & 0 & n.i.^ & 9 A. M.T. & $23 / 09 / 04$ & "target low" & 14 & 0 & n.i. \\
\hline 11 & C. A. & $15 / 07 / 04$ & "target low" & 20 & 0 & n.i. & & $02 / 12 / 04$ & $8,31 \times 10^{2}$ & 18 & 4 & - \\
\hline 12 & A.G. & $30 / 12 / 04$ & "target low" & 2 & 0 & n.i. & & & & & & \\
\hline 13 & N.S. & $18 / 05 / 04$ & n.d. ${ }^{\circ}$ & 1 & 0 & n.i. & & & & & & \\
\hline
\end{tabular}

gio impiegato per la quantificazione e in un caso il campione non è stato sottoposto alla misurazione della carica virale a causa della quantità insufficiente di materiale.

Nell'ambito dei 9 soggetti del gruppo di studio, l'nPCR-RFLP ha identificato in 6 casi $(66.7 \%)$ tutti i codoni virali d'interesse, in 2 casi $(22.2 \%)$ 3 codoni e in 1 caso (11.1\%), infine, negativo all'nPCR-RFLP condotta sul primo campione (concentrazione di DNA virale al di sotto del limite rivelabile dal saggio quantitativo) ha identificato 4 codoni sul campione prelevato a distanza di tre mesi. Di tutti i ceppi identificati nei codoni d'interesse, uno ha dimostrato un profilo di restrizione caratteristico della presenza delle mutazioni M460V e/o M460I ATA-codificata (Tabella II). Questo ceppo è stato rivelato da un soggetto che al momento del prelievo (circa tre mesi dal trapianto di rene) era al termine della profilassi orale con GCV. In questo sogget- to l'antigenemia di HCMV era di 400 PMNL HCMV-pp65 positivi/2 x $10^{5}$, con un aumento di oltre 300 PMNL positivi/ $2 \times 10^{5}$ nell' arco dei 15 giorni precedenti. Il sequenziamento del prodotto di amplificazione di $532 \mathrm{pb}$ contenente il codone 460 mutato ha dimostrato la presenza della mutazione da ATG a GTG corrispondente alla sostituzione aminoacidica da metionina a valina.

\section{DISCUSSIONE}

In questo studio è stato allestito in forma originale un procedimento di nPCR-RFLP per l'identificazione di quattro sequenze nucleotidiche del gene UL97 di HCMV contenenti mutazioni associate a GCV-resistenza. Per la prima volta primer già descritti in letteratura sono stati utilizzati come inneschi di seconda amplificazione. La scelta dell'nPCR rispetto a quella "single step" è stata motivata dal fatto che la prima consente di 
ottenere un maggior numero di copie genomiche del prodotto di amplificazione, necessarie per garantire maggior efficienza dei saggi di RFLP. Inoltre per distinguere le mutazioni M460V/I (ATA) da M460I (ATT) è stata impiegata la combinazione degli enzimi NlaIII e Tru9I su un amplicone di dimensioni diverse rispetto a quello utilizzato dall'autore di riferimento (4).

La sensibilità analitica dell'nPCR, valutata su una singola serie di diluizioni dei due ceppi HCMV-AD169 e HCMV-Towne, ha dimostrato un limite minimo di rilevabilità intorno a 0,13 PFU/reazione.

Dalle prove condotte su ceppi sia "wild-type" sia mutati di riferimento l'nPCR-RFLP, da un lato, si è rivelata di esecuzione relativamente semplice e rapida (il saggio è completato entro due giorni con un intervento manuale di circa tre ore) e, dall'altro, in grado di fornire risultati specifici.

I profili di restrizione delle sequenze "wild-type" per tutti i codoni di interesse e quelli delle sequenze mutate relative ai codoni UL97-460, UL97-594 e UL97-595 si sono dimostrati secondo l'atteso in tutti i campioni di controllo analizzati.

Inoltre il procedimento sembra essere idoneo alla rivelazione di popolazioni virali miste. Lo dimostrerebbero i profili di restrizione ottenuti dai campioni di riferimento contenenti due o più ceppi di HCMV ("Strain pp6": due ceppi UL97460V/I mutati; "Isolat 2": due ceppi UL97460V/I mutati e un ceppo "wild-type").

L'impiego del saggio nPCR-RFLP su 22 soggetti sottoposti a trapianto d'organo o di midollo non ha rivelato ceppi mutati nel gruppo dei soggetti di controllo non trattati (13 soggetti). Al contrario ha rivelato una percentuale relativamente alta, pari all'11\% (1 su 9 soggetti), di ceppi di HCMV con mutazioni associate a farmaco-resistenza nel gruppo dei soggetti di studio sottoposti a profilassi orale con GCV. Sebbene tale percentuale non possa essere confrontata con quelle riportate in letteratura (1) e in particolare con quella del $9,5 \%$ ottenuta in uno studio condotto su una coorte di 200 pazienti sottoposti a trapianto d'organo e a trattamento con GCV (8) a causa del numero particolarmente esiguo dei casi esaminati nel presente studio, é possibile che il ritrovamento di un caso su 9 di infezione con HCMV farmaco-resistente in un soggetto sottoposto a trapianto di rene e a profilassi orale con GCV da tre mesi, rappresenti un dato non trascurabile.

In conclusione, i risultati ottenuti sui ceppi di riferimento sia "wild-type" sia mutati e sui campioni clinici potrebbero favorire l'impiego del procedimento di nPCR-RFLP allestito nell'ambito di questa ricerca per lo studio delle mutazioni sul gene UL97 di HCMV farmaco-associate e proporlo come valido strumento per il controllo dell'insorgenza di HCMV mutanti in soggetti sottoposti a trapianto di rene in corso di profilassi con GCV al fine dell'eventuale adozione di migliori strategie terapeutiche. Dato l'ampio uso anche di VGCV per la profilassi e alla luce della recente scoperta dell'insorgenza di mutazioni sul gene UL97 di HCMV indotte da questo farmaco specialmente in soggetti sottoposti a trapianto allogenico di midollo [9], sarà interessante verificare l'utilità del metodo nell'identificare eventuali mutazioni indotte da VGCV in questa categoria di pazienti.

\section{RINGRAZIAMENTI}

Questo lavoro è stato svolto in parte con fondi di ricerca MIUR (progetto di ricerca scientifica di rilevante interesse nazionale - COFIN 2005, prot. n. 2005069219-001).

\section{BIBLIOGRAFIA}

1. Baldanti F, Lurain N, and Gerna G. 2004. Clinical and biologic aspects of human cytomegalovirus resistance to antiviral drugs. Human Immunol; 65: 403-9.

2. Boivin G, Chou S, Quirk MR, et al. 1996. Detection of ganciclovir resistance mutations quantitation of cytomegalovirus (CMV) DNA in leukocytes of patients with fatal disseminated CMV disease. J Infect Dis; 173: 523-8.

3. Chou S, Erice A, Jordan MC, et al. 1995. Analysis of the UL97 phosphotransferase coding sequence in clinical cytomegalovirus isolates and identification of mutations conferring ganciclovir resistance. J Infect Dis; 171: 576-83.

4. Eckle T, Jahn G, Hamprecht K. 2003. High impact of an expanded restriction fragment length polymorphism assay on detection of ganciclovir-resistant UL97 mutants of human cytomegalovirus. Antimicrob Agents Chemother; 47: 442-3.

5. Erice A 1999. Resistance of human cytomegalovirus to antiviral drugs. Clin Microbiol Rev; 12: 286-97.

6. Hanson MN, Preheim LC, Chou S, et al. 1995. Novel mutation in the UL97 gene of a clinical cytomegalovirus strain conferring resistance to ganciclovir. Antimicrobial Agents Chemother; 39: 1204-5.

7. Limaye AP, Corey L, Koelle DM, Davis CL, Boeckh M. 2000 Emergence of ganciclovir-resistant cytomegalovirus disease among recipients of solidorgan transplants. Lancet; 356: 645-9.

8. Lurain NS, Bhorade SM, Pursell KJ, et al. 2002. Analysis and characterization of antiviral drug-resistant cytomegalovirus isolates from solid organ transplant recipients. J Infect Dis; 186, 760-8.

9. Marfori JE, Exner MM, Marousek GI, Chou S, Drew WL. 2007. Development of new cytomegalovirus UL97 and DNA polymerase mutations conferring drug resistance after valganciclovir therapy in allogeneic stem cell recipients. J Clin Virol,38, 120-5.

10. Prix L, Hamprecht K, Holzhuter B, et al. 1999. Comprehensive restriction analysis of the UL97 
region allows early detection of ganciclovir-resistant human cytomegalovirus in an immunocompromised child. J Infect Dis, 180, 491-5.

11. Singh N. 2001. Preemptive therapy versus universal prophylaxis with ganciclovir for cytomegalovirus in solid organ transplant recipients. Clin Infect Dis, 32, 742-51.

Maria Cristina Medici

Sezione di Microbiologia

Dipartimento di Patologia e Medicina di

Laboratorio

Università degli Studi di Parma

Viale Antonio Gramsci, 14 - 43100 Parma

Tel.: +39-0521988885

Fax +39-0521993620

E-mail: mariacristina.medici@unipr.it 